\title{
FUZZY EQUIVALENCE RELATION, FUZZY CONGRUNCE RELATION AND FUZZY NORMAL SUBGROUPS ON GROUP $G$ OVER $t$-NORMS
}

\author{
RASUL RASULI
}

\begin{abstract}
In this study, by using $t$-norms, fuzzy equivalence relation, fuzzy congrunce relation on group $G$, fuzzy relation of subgroup $H$ of group $G$, fuzzy normal subgroups of fuzzy subgroups, direct product of fuzzy subgroups(normal fuzzy subgroups) are introduced and some the their properties will be discussed. Next by using group homomorphisms, the image and pree image of them will be investigated.
\end{abstract}

\section{INTRODUCTION}

In mathematics and abstract algebra, group theory studies the algebraic structures known as groups. The concept of a group is central to abstract algebra: other well-known algebraic structures, such as rings, fields, and vector spaces, can all be seen as groups endowed with additional operations and axioms. Groups recur throughout mathematics, and the methods of group theory have influenced many parts of algebra. Linear algebraic groups and Lie groups are two branches of group theory that have experienced advances and have become subject areas in their own right. In abstract algebra, a normal subgroup is a subgroup that is invariant under conjugation by members of the group of which it is a part. In other words, a subgroup $H$ of a group $G$ is normal in $G$ if and only if $g H=H g$ for all $g$ in $G$. For centuries probability theory and error calculus have been the only models to treat imprecision and uncertainty. However recently a lot of new models have been introduced for handling incomplete information. The fact that crisp relations fail in interpreting real life phenomenon was first expressed by Poincare [25] in 1902 . Half a century later, Menger [21] addressed the issue raised by Poincare and proposed his Probabilistic relations. According to Menger in order to be in harmony with real life continuum, we should sacrifice transitivity and classical definition of relations should be changed and a probability of being related should be allocated to every pair of points belonging to the universe under consideration. Even after this development there remained a silence regarding re-building a rigorous theory of relations with different probabilities associated with them. Undoubtedly the notion of fuzzy set theory initiated by Zadeh [42] in 1965 in a seminal paper, plays the central role for further development. This notion tries to show that an object corresponds more or less to the particular category we want to assimilate it to; that was how the idea of defining the membership of an element to a set not on the Aristotelian pair $\{0,1\}$ any more but on the continuous interval $[0,1]$ was born. The notion of a fuzzy set is completely nonstatistical in nature and the concept of fuzzy set provides a natural way of dealing with problems in which the source of imprecision is the absence of sharply defined criteria of class membership rather than the presence of random variables. In fact the idea of describing all shades of reality was for long the obsession of some logicians [22, 38]. During last four decades the fuzzy set

2010 Mathematics Subject Classification. 03E72, 15A60, 06B10, 20N25, 20K30, 20K25.

Key words and phrases. Fuzzy set theory, norms, groups, congruence relations, homomorphisms, direct products. 
theory has rapidly developed into an area which scientifically as well as from the application point of view, is recognized as a very valuable contribution to the existing knowledge $[4,5,6,7$, $8,9,10,11,12,13,14,15,16,19,23,24,43,44,41]$. After the emergence of fuzzy set theory in 1965 [42], the simple task of looking at relations as fuzzy sets on the universe $X \times X$ was accomplished in a celebrated paper by Zadeh [40], he introduced the concept of fuzzy relation, defined the notion of equivalence, and gave the concept of fuzzy ordering. Fuzzy relations have broad utility. Compared with crisp relations, they have greater expressive power. They are considered as softer models for expressing the strength of links between elements. Starting in early seventies, fuzzy relations have been defined, investigated, and applied in many different ways e.g., in fuzzy modeling, fuzzy diagnosis, and fuzzy control. They also have applications in fields such as Artificial Intelligence, Psychology, Medicine, Economics, and Sociology. In 1971 the study of fuzzy algebraic structures was started with the introduction of the concept of fuzzy subgroups by Rosenfeld [37]. In fact many basic properties in group theory are found to be carried over to fuzzy groups. In 1979 Anthony and Sherwood [2] redefined a fuzzy subgroup of a group using the concept of triangular norm ( $t$-norm, for short). The notion of fuzzy congruence on a group was introduced by Kroki [18] and the concept of fuzzy quotient group was studied by some authors $[3,17,20,39]$. The author by using norms, investigated some properties of fuzzy algebra $[26,27,28,29,30,31,32,33,34,35,36]$. The purpose of this study is to deal with the algebraic structure of fuzzy equivalence relation, fuzzy congrunce relation on group $G$, fuzzy relation of subgroup $H$ of group $G$, fuzzy normal subgroups of fuzzy subgroups, direct product of fuzzy subgrops(normal fuzzy subgroups) with respet to $t$-norms. In Section 2, we summarize some basic conceps which will be used throughout the paper. In Section 3, by using $t$-norms, we introduse fuzzy equivalence relation and fuzzy congrunce relation on group $G$ and we investigate the intersection of them. Also we define fuzzy relation of subgroup $H$ of group $G$ under $t$-norms and we show that it will be fuzzy equivalence relation on group $G$ under $t$-norms. Later, we define and investigate some properties as intersection and composition of fuzzy subgroups of normal subgroups of group $G$ by using $t$-norms. Also we define normal fuzzy subgroups of group $G$ by using $t$-norms and investigate them. Next we define fuzzy normal subgroups of fuzzy subgroups under $t$-norms and we prove that the intersection of them is also fuzzy normal subgroup with respect to $t$-norms. In Section 4 , we introduce the direct product of fuzzy subgroups (normal fuzzy subgroups) and we prove that it will be fuzzy subgroup(normal fuzzy subgroup) with respect to $t$-norms. In Section 5, we prove that image and pre image of fuzzy subgroups (normal fuzzy subgroups) with respect to $t$-norms is also fuzzy subgroups (normal fuzzy subgroups) under group homomorphisms.

\section{PRELIMINARY}

Definition 2.1. (See $[9,40]$ ) A fuzzy subset of $G$, we mean a function $\mu: G \rightarrow[0,1]$. The set of all fuzzy subsets of $G$ is called the $[0,1]$-power set of $G$ and is denoted $[0,1]^{G}$. By a fuzzy relation on $G$ we mean a function $\mu: G \times G \rightarrow[0,1]$. Denote by $F R(G)$, the set of all fuzzy relations on $G$.

Definition 2.2. (See $[9,40])$ Let $\mu_{1}, \mu_{2} \in F R(G)$ and $x, y \in G$. We define

(1) $\mu_{1} \subseteq \mu_{2}$ iff $\mu_{1}(x, y) \leq \mu_{2}(x, y)$,

(2) $\mu_{1}=\mu_{2}$ iff $\mu_{1}(x, y)=\mu_{2}(x, y)$.

Definition 2.3. (See [1]) A $t$-norm $T$ is a function $T:[0,1] \times[0,1] \rightarrow[0,1]$ having the following four properties: 
(T1) $T(x, 1)=x$ (neutral element),

(T2) $T(x, y) \leq T(x, z)$ if $y \leq z$ (monotonicity),

(T3) $T(x, y)=T(y, x)$ (commutativity),

(T4) $T(x, T(y, z))=T(T(x, y), z)$ (associativity), for all $x, y, z \in[0,1]$.

We say that $T$ is idempotent if for all $x \in[0,1], T(x, x)=x$.

Example 2.4. (1) Standard intersection $T$-norm $T_{m}(x, y)=\min \{x, y\}$.

(2) Bounded sum $T$-norm $T_{b}(x, y)=\max \{0, x+y-1\}$.

(3) algebraic product $T$-norm $T_{p}(x, y)=x y$.

(4) Drastic $T$-norm

$$
T_{D}(x, y)= \begin{cases}y & \text { if } x=1 \\ x & \text { if } y=1 \\ 0 & \text { otherwise }\end{cases}
$$

(5) Nilpotent minimum $T$-norm

$$
T_{n M}(x, y)=\left\{\begin{aligned}
\min \{x, y\} & \text { if } x+y>1 \\
0 & \text { otherwise. }
\end{aligned}\right.
$$

(6) Hamacher product $T$-norm

$$
T_{H_{0}}(x, y)=\left\{\begin{aligned}
0 & \text { if } x=y=0 \\
\frac{x y}{x+y-x y} & \text { otherwise. }
\end{aligned}\right.
$$

The drastic $t$-norm is the pointwise smallest $t$-norm and the minimum is the pointwise largest $t$-norm: $T_{D}(x, y) \leq T(x, y) \leq T_{\min }(x, y)$ for all $x, y \in[0,1]$.

Lemma 2.5. (See [1]) Let $T$ be a t-norm. Then

$$
T(T(x, y), T(w, z))=T(T(x, w), T(y, z)),
$$

for all $x, y, w, z \in[0,1]$.

Definition 2.6. (See 36]) Let $\mu$ be a fuzzy subset of a group $G$. Then $\mu$ is called a fuzzy subgroup of $G$ under a $t$-norm $T$ ( $T$-fuzzy subgroup) iff for all $x, y \in G$

(1) $\mu(x y) \geq T(\mu(x), \mu(y))$

(2) $\mu\left(x^{-1}\right) \geq \mu(x)$.

Denote by $T F(G)$, the set of all $T$-fuzzy subgroup of $G$.

Example 2.7. Let $G=\{1, i,-1,-i\}$ be a group with respect to multiplication. Define fuzzy subset $\mu: G \rightarrow[0,1]$ as

$$
\mu(x)= \begin{cases}0.9 & \text { if } x=1 \\ 0.8 & \text { if } x=-1 \\ 0.6 & \text { if } x= \pm i\end{cases}
$$

If $T(x, y)=T_{m}(x, y)=\min \{x, y\}$ for all $x, y \in G$, then $\mu \in T F(G)$. 
Definition 2.8. (See [36]) Let $f$ be a mapping from $\frac{G_{1}}{H_{1}}$ into $\frac{G_{2}}{H_{2}}, \mu \in[0,1]^{\frac{G_{1}}{H_{1}}}$ and $\nu \in[0,1]^{\frac{G_{2}}{H_{2}}}$. Define $f(\mu) \in[0,1]^{\frac{G_{2}}{H_{2}}}$ as

$$
f(\mu)\left(g_{2} H_{2}\right)=\left\{\begin{aligned}
\sup \left\{\mu\left(g_{1} H_{1}\right) \mid g_{1} H_{1} \in \frac{G_{1}}{H_{1}}, f\left(g_{1} H_{1}\right)=g_{2} H_{2}\right\} & \text { if } f^{-1}\left(g_{2} H_{2}\right) \neq \emptyset \\
0 & \text { if } f^{-1}\left(g_{2} H_{2}\right)=\emptyset
\end{aligned}\right.
$$

for all $g_{2} H_{2} \in G_{2} / H_{2}$.

Also define $f^{-1}(\nu) \in[0,1]^{\frac{G_{1}}{H_{1}}}$ by $f^{-1}(\nu)\left(g_{1} H_{1}\right)=\nu\left(f\left(g_{1} H_{1}\right)\right)$ for all $g_{1} H_{1} \in G_{1} / H_{1}$.

Definition 2.9. (See [36]) Given two groups $G$ and $H$, a group homomorphism is a map $f: G \rightarrow H$ such that $f(x y)=f(x) f(y)$ for all $x, y \in G$.

Example 2.10. The map exp $:(\mathbb{R},+) \rightarrow(\mathbb{R},$.$) with \exp (x)=e^{x}$ is a group homomorphism.

Definition 2.11. (See [36]) Let $H$ be normal subgroup of $G$. The group homomorphism $\pi$ : $G \rightarrow \frac{G}{H}$ with $\pi(g)=g H$ is called the natural or canonical map or projection.

Remark 2.12. (See [36]) We have the following terminology: epimorphism=surjective homomorphism.

\section{FUZZY EQUIVALENCE RELATION, FUZZY CONGRUNCE RELATION, FUZZY QUOTIENT SUBGROUPS}

AND NORMAL FUZZY QUOTIENT SUBGROUPS WITH RESPECT TO A $t$-NORM

Throughout this Section $G$ be an arbitrary group. In this study we define some new special $T$-fuzzy equivalence relations and derive some simple consequences. Then using those relations we define suitable $T$-fuzzy quotient subgroup of $\frac{G}{H}$ differently.

Definition 3.1. A fuzzy relation $\mu: G \times G \rightarrow[0,1]$ on a group $G$ is a $T$-fuzzy equivalence relation on $G$ if the following conditions are satisfied:

(1) $\mu(x, x)=1$.

(2) $\mu(x, y)=\mu(y, x)$.

(3) $\mu(x, z) \geq T(\mu(x, y), \mu(y, z))$,

for all $x, y, z \in G$.

Example 3.2. Let $G=(\mathbb{Z},+)$ be a group of integers numbers. Define $\mu: \mathbb{Z} \times \mathbb{Z} \rightarrow[0,1]$ by

$$
\mu(x, y)=\left\{\begin{aligned}
1 & \text { if } x=y \\
0.55 & \text { otherwise. }
\end{aligned}\right.
$$

If $T(x, y)=T_{m}(x, y)=\min \{x, y\}$ for all $x, y \in[0,1]$, Then $\mu$ is a $T$-fuzzy equivalence relation on $G$.

Definition 3.3. $\mu_{1}$ and $\mu_{2}$ be two $T$-fuzzy equivalence relations on $G$. We define

$$
\left(\mu_{1} \cap \mu_{2}\right)(x, y)=T\left(\mu_{1}(x, y), \mu_{2}(x, y)\right)
$$

for all $x, y \in G$.

Remark 3.4. $\mu_{1}$ and $\mu_{2}$ and $\mu_{3}$ be three $T$-fuzzy equivalence relations on $G$. Then from properties T3 and T4 of Definition 2.3 we get that $\mu_{1} \cap \mu_{2}=\mu_{2} \cap \mu_{1}$ and $\mu_{1} \cap \mu_{2} \cap \mu_{3}=\left(\mu_{1} \cap \mu_{2}\right) \cap \mu_{3}=$ $\mu_{1} \cap\left(\mu_{2} \cap \mu_{3}\right)$. 
Proposition 3.5. If $\mu_{1}$ and $\mu_{2}$ be two $T$-fuzzy equivalence relations on $G$, then so is $\mu_{1} \cap \mu_{2}$.

Proof. Let $x, y, z \in G$.

(1) $\left(\mu_{1} \cap \mu_{2}\right)(x, x)=T\left(\mu_{1}(x, x), \mu_{2}(x, x)\right)=T(1,1)=1$.

(2) $\left(\mu_{1} \cap \mu_{2}\right)(x, y)=T\left(\mu_{1}(x, y), \mu_{2}(x, y)\right)=T\left(\mu_{1}(y, x), \mu_{2}(y, x)\right)=\left(\mu_{1} \cap \mu_{2}\right)(y, x)$.

$(3)$

$$
\begin{gathered}
\left(\mu_{1} \cap \mu_{2}\right)(x, y)=T\left(\mu_{1}(x, y), \mu_{2}(x, y)\right) \\
\geq T\left(T\left(\mu_{1}(x, z), \mu_{1}(z, y)\right), T\left(\mu_{2}(x, z), \mu_{2}(z, y)\right)\right. \\
=T\left(T\left(\mu_{1}(x, z), \mu_{2}(x, z)\right), T\left(\mu_{1}(z, y), \mu_{2}(z, y)\right)\right) \quad(\text { by Lemma } 2.5) \\
=T\left(\left(\mu_{1} \cap \mu_{2}\right)(x, z),\left(\mu_{1} \cap \mu_{2}\right)(z, y)\right) .
\end{gathered}
$$

Thus $\mu_{1} \cap \mu_{2}$ will be $T$-fuzzy equivalence relations on $G$.

Corollary 3.6. Let $I_{n}=\{1,2, \ldots, n\}$. If $\left\{\mu_{i} \mid i \in I_{n}\right\}$ be $T$-fuzzy equivalence relations on $G$, then so is $\mu=\cap_{i \in I_{n}} \mu_{i}$.

Definition 3.7. A fuzzy relation $\mu: G \times G \rightarrow[0,1]$ on a group $G$ is a $T$-fuzzy congruence relation on $G$ if the following conditions are satisfied:

(1) $\mu(x, x)=1$.

(2) $\mu(x, y)=\mu(y, x)$.

(3) $\mu(x, z) \geq T(\mu(x, y), \mu(y, z))$.

(4) $\mu(x z, y t) \geq T(\mu(x, y), \mu(z, t))$, for all $x, y, z, t \in G$.

Example 3.8. Let $G=(\mathbb{R},+)$ be a group of real numbers. Define $\mu: \mathbb{R} \times \mathbb{R} \rightarrow[0,1]$ by

$$
\mu(x, y)=\left\{\begin{aligned}
1 & \text { if } x=y \\
0.35 & \text { otherwise. }
\end{aligned}\right.
$$

If $T(x, y)=T_{m}(x, y)=\min \{x, y\}$ for all $x, y \in[0,1]$, Then $\mu$ is a $T$-fuzzy congruence relation on $G$.

Proposition 3.9. If $\mu_{1}$ and $\mu_{2}$ be two T-fuzzy congruence relations on $G$, then so is $\mu_{1} \cap \mu_{2}$.

Proof. Let $x, y, z, t \in G$. Then

(1) $\left(\mu_{1} \cap \mu_{2}\right)(x, x)=T\left(\mu_{1}(x, x), \mu_{2}(x, x)\right)=T(1,1)=1$.

(2) $\left(\mu_{1} \cap \mu_{2}\right)(x, y)=T\left(\mu_{1}(x, y), \mu_{2}(x, y)\right)=T\left(\mu_{1}(y, x), \mu_{2}(y, x)\right)=\left(\mu_{1} \cap \mu_{2}\right)(y, x)$.

(3)

$$
\begin{gathered}
\left(\mu_{1} \cap \mu_{2}\right)(x, y)=T\left(\mu_{1}(x, y), \mu_{2}(x, y)\right) \\
\geq T\left(T\left(\mu_{1}(x, z), \mu_{1}(z, y)\right), T\left(\mu_{2}(x, z), \mu_{2}(z, y)\right)\right. \\
=T\left(T\left(\mu_{1}(x, z), \mu_{2}(x, z)\right), T\left(\mu_{1}(z, y), \mu_{2}(z, y)\right)\right) \quad(\text { by Lemma } 2.5) \\
=T\left(\left(\mu_{1} \cap \mu_{2}\right)(x, z),\left(\mu_{1} \cap \mu_{2}\right)(z, y)\right) .
\end{gathered}
$$

(4)

$$
\begin{gathered}
\left(\mu_{1} \cap \mu_{2}\right)(x z, y t)=T\left(\mu_{1}(x z, y t), \mu_{2}(x z, y t)\right) \\
\geq T\left(T\left(\mu_{1}(x, y), \mu_{1}(z, t)\right), T\left(\mu_{2}(x, y), \mu_{2}(z, t)\right)\right) \\
=T\left(T\left(\mu_{1}(x, y), \mu_{2}(x, y)\right), T\left(\mu_{1}(z, t), \mu_{2}(z, t)\right)\right) \quad(\text { by Lemma } 2.5) \\
=T\left(\left(\mu_{1} \cap \mu_{2}\right)(x, y),\left(\mu_{1} \cap \mu_{2}\right)(z, t)\right) .
\end{gathered}
$$

Then $\mu_{1} \cap \mu_{2}$ is $T$-fuzzy congruence relation on $G$. 
Corollary 3.10. Let $I_{n}=\{1,2, \ldots, n\}$. If $\left\{\mu_{i} \mid i \in I_{n}\right\}$ be $T$-fuzzy congruence relations on $G$, then so is $\mu=\cap_{i \in I_{n}} \mu_{i}$.

We define some special fuzzy relations and give some its results.

Definition 3.11. Let $H$ be a subgroup of $G$ and $\mu_{H} \in T F(H)$. A $T$-fuzzy relation $\theta: G \times G \rightarrow$ $[0,1]$ can be defined by

$$
\theta(x, y)=\left\{\begin{aligned}
T\left(\mu_{H}(x), \mu_{H}(y)\right) & \text { if } x \neq y \\
\mu_{H}(e)=1 & \text { if } x=y
\end{aligned}\right.
$$

Corollary 3.12. $\theta\left(x^{-1}, y^{-1}\right) \geq \theta(x, y)$ for all $x, y \in G$.

Proof. Since $\mu_{H} \in T F(H)$ so

$$
\theta\left(x^{-1}, y^{-1}\right)=T\left(\mu_{H}\left(x^{-1}\right), \mu_{H}\left(y^{-1}\right)\right) \geq T\left(\mu_{H}(x), \mu_{H}(y)\right)=\theta(x, y) .
$$

Proposition 3.13. $\theta$ is a T-fuzzy equivalence relation on $G$.

Proof. Let $x, y, z \in G$, then

(1) $\theta(x, x)=1$.

(2)

$$
\theta(x, y)=T\left(\mu_{H}(x), \mu_{H}(y)\right)=T\left(\mu_{H}(y), \mu_{H}(x)\right)=\theta(y, x)
$$

$$
\begin{gathered}
\theta(x, y)=T(\theta(x, y), 1)=T(\theta(x, y), \theta(z, z)) \\
=T\left(T\left(\mu_{H}(x), \mu_{H}(y)\right), T\left(\mu_{H}(z), \mu_{H}(z)\right)\right) \\
=T\left(T\left(\mu_{H}(x), \mu_{H}(z)\right), T\left(\mu_{H}(y), \mu_{H}(z)\right)\right) \quad(\text { by Lemma 2.5) } \\
=T(\theta(x, z), \theta(z, y)) .
\end{gathered}
$$

Then $\theta$ is a $T$-fuzzy equivalence relation on $G$.

Proposition 3.14. The T-fuzzy relation $\theta$ defined on $G$ is T-fuzzy congruence relation.

Proof. Let $x, y, z, t \in G$ and $\mu_{H} \in T F(H)$. Then

(1) $\theta(x, x)=1$.

(2)

$$
\theta(x, y)=T\left(\mu_{H}(x), \mu_{H}(y)\right)=T\left(\mu_{H}(y), \mu_{H}(x)\right)=\theta(y, x)
$$

$$
\begin{gathered}
\theta(x, y)=T(\theta(x, y), 1)=T(\theta(x, y), \theta(z, z)) \\
=T\left(T\left(\mu_{H}(x), \mu_{H}(y)\right), T\left(\mu_{H}(z), \mu_{H}(z)\right)\right) \\
=T\left(T\left(\mu_{H}(x), \mu_{H}(z)\right), T\left(\mu_{H}(y), \mu_{H}(z)\right)\right) \quad(\text { by Lemma 2.5) } \\
=T(\theta(x, z), \theta(z, y)) .
\end{gathered}
$$

(4)

$$
\begin{gathered}
\theta(x z, y t)=T\left(\mu_{H}(x z), \mu_{H}(y t)\right) \\
\geq T\left(T\left(\mu_{H}(x), \mu_{H}(z)\right), T\left(\mu_{H}(y), \mu_{H}(t)\right)\right) \\
=T\left(T\left(\mu_{H}(x), \mu_{H}(y)\right), T\left(\mu_{H}(z), \mu_{H}(t)\right)\right) \quad(\text { by Lemma 2.5) }
\end{gathered}
$$


FUZZY EQUIVALENCE RELATION, FUZZY CONGRUNCE RELATION AND FUZZY NORMAL SUBGROUPS ON GROUP $G$ OVER

$$
=T(\theta(x, y), \theta(z, t))
$$

Thus $\theta$ will be $T$-fuzzy congruence relation on $G$.

Definition 3.15. Let $G$ be a group and $H$ be a normal subgroup of $G$. Then $\mu_{\frac{G}{H}}: \frac{G}{H} \rightarrow[0,1]$ can be defined by $\mu_{\frac{G}{H}}(x H)=\theta(x, h)$ for all $x \in G$ and $h \in H$.

Now we show some algebraic properties of $\mu$.

Lemma 3.16. Let $\mu_{H} \in T F(H)$ and $T$ be idempotent t-norm. Then $\mu_{\frac{G}{H}} \in T F\left(\frac{G}{H}\right)$.

Proof. Let $x H, y H \in \frac{G}{H}$ and $\mu_{H} \in T F(H)$. Then

$$
\begin{gathered}
\mu_{\frac{G}{H}}(x H y H)=\mu_{\frac{G}{H}}(x y H)=\theta(x y, h) \\
=T\left(\mu_{H}(x y), \mu_{H}(h)\right) \geq T\left(T\left(\mu_{H}(x), \mu_{H}(y)\right), \mu_{H}(h)\right) \\
=T\left(T\left(\mu_{H}(x), \mu_{H}(y)\right), T\left(\mu_{H}(h), \mu_{H}(h)\right)\right) \\
=T\left(T\left(\mu_{H}(x), \mu_{H}(h)\right), T\left(\mu_{H}(y), \mu_{H}(h)\right)\right) \quad(\text { by Lemma 2.5) } \\
=T(\theta(x, h), \theta(y, h))=T\left(\mu_{\frac{G}{H}}(x H), \mu_{\frac{G}{H}}(y H)\right) .
\end{gathered}
$$

Also

$$
\begin{gathered}
\mu_{\frac{G}{H}}(x H)^{-1}=\mu_{\frac{G}{H}}\left(x^{-1} H\right)=\theta\left(x^{-1}, h\right) \\
=T\left(\mu_{H}\left(x^{-1}\right), \mu_{H}(h)\right) \geq T\left(\mu_{H}(x), \mu_{H}(h)\right) \\
=\theta(x, h)=\mu_{\frac{G}{H}}(x H) .
\end{gathered}
$$

Therefore $\mu_{\frac{G}{H}} \in T F\left(\frac{G}{H}\right)$.

Proposition 3.17. If $T$ be idempotent -norm, then for all $x H \in \frac{G}{H}$, and $n \geq 1$,

(1) $\mu_{\frac{G}{H}}(H) \geq \mu_{\frac{G}{H}}(x H)$;

(2) $\mu_{\frac{G}{H}}(x H)^{n} \geq \mu_{\frac{G}{H}}(x H)$;

(3) $\mu_{\frac{G}{H}}(x H)=\mu_{\frac{G}{H}}(x H)^{-1}$.

Proof. Let $x H \in \frac{G}{H}$ and $n \geq 1$. From Lemma 3.16 we have that $\mu_{\frac{G}{H}} \in T F\left(\frac{G}{H}\right)$.

$$
\begin{gathered}
\mu_{\frac{G}{H}}(H)=\mu_{\frac{G}{H}}\left(x x^{-1} H\right)=\mu_{\frac{G}{H}}\left(x H x^{-1} H\right) \\
\geq T\left(\mu_{\frac{G}{H}}(x H), \mu_{\frac{G}{H}}\left(x^{-1} H\right)\right) \geq T\left(\mu_{\frac{G}{H}}(x H), \mu_{\frac{G}{H}}(x H)\right) \\
=\mu_{\frac{G}{H}}(x H) .
\end{gathered}
$$

(2) $\mu_{\frac{G}{H}}(x H)^{n}=\mu_{\frac{G}{H}}(\underbrace{x H x H \ldots x H}_{n}) \geq T(\underbrace{\mu_{\frac{G}{H}}(x H), \mu_{\frac{G}{H}}(x H), \ldots, \mu_{\frac{G}{H}}(x H)}_{n})=\mu_{\frac{G}{H}}(x H)$.

(3) $\mu_{\frac{G}{H}}(x H)=\mu_{\frac{G}{H}}\left(\left(x^{-1} H\right)\right)^{-1} \geq \mu_{\frac{G}{H}}\left(x^{-1} H\right) \geq \mu_{\frac{G}{H}}(x H)$. So $\mu_{\frac{G}{H}}(x H)=\mu_{\frac{G}{H}}\left(x^{-1} H\right)$.

Proposition 3.18. Let $\mu_{\frac{G}{H}}$ be a fuzzy subset of a finite group $\frac{G}{H}$ and $T$ be idempotent t-norm. If $\mu_{\frac{G}{H}}$ satisfies condition (1) of Definition 2.6, then $\mu_{\frac{G}{H}} \in T F\left(\frac{G}{H}\right)$. 
Proof. Let $x H \in \frac{G}{H}, x \notin H$. Since $\frac{G}{H}$ is finite, $x H$ has finite order, say $n>1$. So $(x H)^{n}=H$ and $x^{-1} H=x^{n-1} H$. Now by using (1) repeatedly, we have that

$$
\begin{gathered}
\mu_{\frac{G}{H}}\left(x^{-1} H\right)=\mu_{\frac{G}{H}}\left(x^{n-1} H\right)=\mu_{\frac{G}{H}}\left(x^{n-2} x H\right) \\
\geq T\left(\mu_{\frac{G}{H}}\left(x^{n-2} H\right), \mu_{\frac{G}{H}}(x H)\right) \geq T \underbrace{\mu_{\frac{G}{H}}(x H), \mu_{\frac{G}{H}}(x H), \ldots, \mu_{\frac{G}{H}}(x H)}_{n}) \\
=\mu_{\frac{G}{H}}(x H) .
\end{gathered}
$$

Proposition 3.19. Let $\mu_{\frac{G}{H}}, \nu_{\frac{G}{H}} \in T F\left(\frac{G}{H}\right)$. Then $\mu_{\frac{G}{H}} \cap \nu_{\frac{G}{H}} \in T F\left(\frac{G}{H}\right)$.

Proof. Let $x H, y H \in \frac{G}{H}$. Then

$$
\begin{gathered}
\left(\mu_{\frac{G}{H}} \cap \nu_{\frac{G}{H}}\right)(x H y H)=T\left(\mu_{\frac{G}{H}}(x H y H), \nu_{\frac{G}{H}}(x H y H)\right) \\
\geq T\left(T\left(\mu_{\frac{G}{H}}(x H), \mu_{\frac{G}{H}}(y H)\right), T\left(\nu_{\frac{G}{H}}(x H), \nu_{\frac{G}{H}}(y H)\right)\right) \\
=T\left(T\left(\mu_{\frac{G}{H}}(x H), \nu_{\frac{G}{H}}(x H)\right), T\left(\mu_{\frac{G}{H}}(y H), \nu_{\frac{G}{H}}(y H)\right)\right) \quad(\text { by Lemma 2.5) } \\
=T\left(\left(\mu_{\frac{G}{H}} \cap \nu_{\frac{G}{H}}\right)(x H),\left(\mu_{\frac{G}{H}} \cap \nu_{\frac{G}{H}}\right)(y H)\right) .
\end{gathered}
$$

Also

$$
\begin{gathered}
\left(\mu_{\frac{G}{H}} \cap \nu_{\frac{G}{H}}\right)(x H)^{-1}=\left(\mu_{\frac{G}{H}} \cap \nu_{\frac{G}{H}}\right)\left(x^{-1} H\right) \\
=T\left(\mu_{\frac{G}{H}}\left(x^{-1} H\right), \nu_{\frac{G}{H}}\left(x^{-1} H\right)\right) \geq T\left(\mu_{\frac{G}{H}}(x H), \nu_{\frac{G}{H}}(x H)\right) \\
=\left(\mu_{\frac{G}{H}} \cap \nu_{\frac{G}{H}}\right)(x H) .
\end{gathered}
$$

Thus $\mu_{\frac{G}{H}} \cap \nu_{\frac{G}{H}} \in T F\left(\frac{G}{H}\right)$.

Corollary 3.20. Let $I_{n}=\{1,2, \ldots, n\}$. If $\left\{\mu_{i} \mid i \in I_{n}\right\} \subseteq T F\left(\frac{G}{H}\right)$, Then $\mu=\cap_{i \in I_{n}} \mu_{i} \in T F\left(\frac{G}{H}\right)$.

Proposition 3.21. Let $\mu_{\frac{G}{H}} \in T F\left(\frac{G}{H}\right)$ and $x H \in \frac{G}{H}$. If $T$ be idempotent t-norm, then $\mu_{\frac{G}{H}}(x H y H)=\mu_{\frac{G}{H}}(y H)$ for all $y H \in \frac{G}{H}$ if and only if $\mu_{\frac{G}{H}}(x H)=\mu_{\frac{G}{H}}(H)$.

Proof. Suppose that $\mu_{\frac{G}{H}}(x H y H)=\mu_{\frac{G}{H}}(y H)$ for all $y H \in \frac{G}{H}$. Then by letting $y=H$, we get that $\mu_{\frac{G}{H}}(x H)=\mu_{\frac{G}{H}}(H)$.

Conversely, suppose that $\mu_{\frac{G}{H}}(x H)=\mu_{\frac{G}{H}}(H)$. By Proposition 3.17 we have that $\mu_{\frac{G}{H}}(x H) \geq$ $\mu_{\frac{G}{H}}(x H y H), \mu_{\frac{G}{H}}(y H)$. Now we have

$$
\begin{gathered}
\mu_{\frac{G}{H}}(x H y H) \geq T\left(\mu_{\frac{G}{H}}(x H), \mu_{\frac{G}{H}}(y H)\right) \geq T\left(\mu_{\frac{G}{H}}(y H), \mu_{\frac{G}{H}}(y H)\right) \\
=\mu_{\frac{G}{H}}(y H)=\mu_{\frac{G}{H}}\left(x^{-1} x y H\right)=\mu_{\frac{G}{H}}\left(x^{-1} H x H y H\right) \\
\geq T\left(\mu_{\frac{G}{H}}\left(x^{-1} H\right), \mu_{\frac{G}{H}}(x H y H)\right) \geq T\left(\mu_{\frac{G}{H}}(x H), \mu_{\frac{G}{H}}(x H y H)\right) \\
\geq T\left(\mu_{\frac{G}{H}}(x H y H), \mu_{\frac{G}{H}}(x H y H)\right)=\mu_{\frac{G}{H}}(x H y H) .
\end{gathered}
$$

Then $\mu_{\frac{G}{H}}(x H)=\mu_{\frac{G}{H}}(H)$. 
FUZZY EQUIVALENCE RELATION, FUZZY CONGRUNCE RELATION AND FUZZY NORMAL SUBGROUPS ON GROUP $G$ OVER

Definition 3.22. Let $\frac{G}{H}$ be a quotient subgroup and let $\mu_{\frac{G}{H}}, \nu_{\frac{G}{H}}: G / H \rightarrow[0,1]$ be two fuzzy sets in $\frac{G}{H}$. The composition $\mu_{\frac{G}{H}} O \nu_{\frac{G}{H}}: \frac{G}{H} \rightarrow[0,1]$ is defined by

$$
\left(\mu_{\frac{G}{H}} O \nu_{\frac{G}{H}}\right)(x H)=\left\{\begin{aligned}
\sup _{a H b H=x H} T\left(\mu_{\frac{G}{H}}(a H), \nu_{\frac{G}{H}}(b H)\right) & \text { if } a H b H=x H \\
0 & \text { if } a H b H \neq x H
\end{aligned}\right.
$$

Proposition 3.23. Let $\mu_{\frac{G}{H}}^{-1}$ be the inverse of $\mu_{\frac{G}{H}}$ such that $\mu_{G / H}^{-1}(x H)=\mu_{\frac{G}{H}}\left(x^{-1} H\right)$. Then $\mu_{\frac{G}{H}} \in T F\left(\frac{G}{H}\right)$ if and only if $\mu_{\frac{G}{H}}$ satisfies the following conditions:

(1) $\mu_{\frac{G}{H}} O \mu_{\frac{G}{H}} \subseteq \mu_{\frac{G}{H}}$;

(2) $\mu_{\frac{G}{H}}^{-1}=\mu_{\frac{G}{H}}$.

Proof. Let $x H, y H, z H \in \frac{G}{H}$ such that $x H=y H z H$. If $\mu_{\frac{G}{H}} \in T F\left(\frac{G}{H}\right)$, then

$$
\mu_{\frac{G}{H}}(x H)=\mu_{\frac{G}{H}}(y H z H) \geq T\left(\mu_{\frac{G}{H}}(y H), \mu_{\frac{G}{H}}(z H)\right)=\left(\mu_{\frac{G}{H}} O \mu_{\frac{G}{H}}\right)(x H)
$$

so $\mu_{\frac{G}{H}} O \mu_{\frac{G}{H}} \subseteq \mu_{\frac{G}{H}}$. Also by Proposition 3.17 we have $\mu_{\frac{G}{H}}^{-1}(x H)=\mu_{\frac{G}{H}}\left(x^{-1} H\right)=\mu_{\frac{G}{H}}(x H)$.

Conversely, let $x H \in \frac{G}{H}$. Then

$$
\begin{gathered}
\mu_{\frac{G}{H}}(y H z H)=\mu_{\frac{G}{H}}(x H) \geq\left(\mu_{\frac{G}{H}} O \mu_{\frac{G}{H}}\right)(x H) \\
=\sup _{x H=y H z H} T\left(\mu_{\frac{G}{H}}(y H), \mu_{\frac{G}{H}}(z H)\right) \geq T\left(\mu_{\frac{G}{H}}(y H), \mu_{\frac{G}{H}}(z H)\right) .
\end{gathered}
$$

Definition 3.24. We say that $\mu \in T F(G)$ is a normal $T$-fuzzy subgroup of $G$ if for all $x, y \in G$, $\mu\left(x y x^{-1}\right)=\mu(y)$. Also we denote by $N T F(G)$ the set of all normal $T$-fuzzy subgroups of $G$.

Corollary 3.25. If $\mu_{H} \in N T F(H)$, then $\mu_{\frac{G}{H}} \in N T F\left(\frac{G}{H}\right)$.

Proof. Let $x H, y H \in \frac{G}{H}$ and $\mu_{H} \in N T F(H)$. Then

$$
\begin{gathered}
\mu_{\frac{G}{H}}\left(x H y H(x H)^{-1}\right)=\mu_{\frac{G}{H}}\left(x H y H x^{-1} H\right)=\mu_{\frac{G}{H}}\left(x y x^{-1} H\right) \\
=\theta\left(x y x^{-1}, h\right)=T\left(\mu_{H}\left(x y x^{-1}\right), \mu_{H}(h)\right) \\
=T\left(\mu_{H}(y), \mu_{H}(h)\right)=\theta(y, h)=\mu_{\frac{G}{H}}(y H) .
\end{gathered}
$$

Proposition 3.26. Let $\mu_{\frac{G}{H}}, \nu_{\frac{G}{H}} \in N T F\left(\frac{G}{H}\right)$. Then $\mu_{\frac{G}{H}} \cap \nu_{\frac{G}{H}} \in N T F\left(\frac{G}{H}\right)$.

Proof. Let $x H, y H \in \frac{G}{H}$. Then

$$
\begin{gathered}
\left(\mu_{\frac{G}{H}} \cap \nu_{\frac{G}{H}}\right)\left(x H y H(x H)^{-1}\right)=\left(\mu_{\frac{G}{H}} \cap \nu_{\frac{G}{H}}\right)\left(x H y H x^{-1} H\right) \\
=T\left(\mu_{\frac{G}{H}}\left(x H y H x^{-1} H\right), \nu_{\frac{G}{H}}\left(x H y H x^{-1} H\right)\right)=T\left(\mu_{\frac{G}{H}}(y H), \nu_{\frac{G}{H}}(y H)\right) \\
=\left(\mu_{\frac{G}{H}} \cap \nu_{\frac{G}{H}}\right)(y H) .
\end{gathered}
$$

Corollary 3.27. Let $I_{n}=\{1,2, \ldots, n\}$. If $\left\{\mu_{i} \mid i \in I_{n}\right\} \subseteq N T F\left(\frac{G}{H}\right)$, Then $\mu=\cap_{i \in I_{n}} \mu_{i} \in$ $\operatorname{NTF}\left(\frac{G}{H}\right)$. 
Definition 3.28. Let $\mu_{\frac{G}{H}}, \nu_{\frac{G}{H}} \in T F\left(\frac{G}{H}\right)$ and $\mu_{\frac{G}{H}} \subseteq \nu_{\frac{G}{H}}$. Then $\mu_{\frac{G}{H}}$ is called a normal subgroup of the subgroup $\nu_{\frac{G}{H}}$, written $\mu_{\frac{G}{H}} \unrhd \nu_{\frac{G}{H}}$, if for all $x H, y H \in \frac{G}{H}$ we have that

$$
\mu_{\frac{G}{H}}\left(x H y H(x H)^{-1}\right) \geq T\left(\mu_{\frac{G}{H}}(y H), \nu_{\frac{G}{H}}(x H)\right) .
$$

Lemma 3.29. Let $T$ be idempotent t-norm. If $\mu_{\frac{G}{H}} \in N T F\left(\frac{G}{H}\right)$ and $\nu_{\frac{G}{H}} \in T F\left(\frac{G}{H}\right)$, then $\mu_{\frac{G}{H}} \cap$ $\nu_{\frac{G}{H}} \unrhd \nu_{\frac{G}{H}}$.

Proof. From Proposition 3.19 we have $\left(\mu_{\frac{G}{H}} \cap \nu_{\frac{G}{H}}\right) \in T F\left(\frac{G}{H}\right)$. Now for all $x H, y H \in \frac{G}{H}$ we have

$$
\begin{gathered}
\left(\mu_{\frac{G}{H}} \cap \nu_{\frac{G}{H}}\right)\left(x H y H(x H)^{-1}\right) \\
=T\left(\mu_{\frac{G}{H}}\left(x H y H(x H)^{-1}\right), \nu_{\frac{G}{H}}\left(x H y H(x H)^{-1}\right)\right) \\
=T\left(\mu_{\frac{G}{H}}(y H), \nu_{\frac{G}{H}}\left(x H y H(x H)^{-1}\right)\right) \\
\geq T\left(\mu_{\frac{G}{H}}(y H), T\left(T\left(\nu_{\frac{G}{H}}(x H), \nu_{\frac{G}{H}}(y H)\right), \nu_{\frac{G}{H}}(x H)\right)\right) \\
=T\left(\mu_{\frac{G}{H}}(y H), T\left(\nu_{\frac{G}{H}}(y H), \nu_{\frac{G}{H}}(x H)\right)\right) \\
=T\left(T\left(\mu_{\frac{G}{H}}(y H), \nu_{\frac{G}{H}}(y H)\right), \nu_{\frac{G}{H}}(x H)\right) \\
=T\left(\left(\mu_{\frac{G}{H}} \cap \nu_{\frac{G}{H}}\right)(y H), \nu_{\frac{G}{H}}(x H)\right) .
\end{gathered}
$$

Hence $\mu_{\frac{G}{H}} \cap \nu_{\frac{G}{H}} \unrhd \nu_{\frac{G}{H}}$.

Proposition 3.30. Let $T$ be idempotent and $\mu_{\frac{G}{H}}, \mu_{\frac{G}{H}}, \xi_{\frac{G}{H}} \in T F\left(\frac{G}{H}\right)$. If $\mu_{\frac{G}{H}}, \nu_{\frac{G}{H}} \unrhd \xi_{\frac{G}{H}}$, then $\mu_{\frac{G}{H}} \cap \nu_{\frac{G}{H}} \unrhd \xi_{\frac{G}{H}}$.

Proof. Clearly, $\mu_{\frac{G}{H}} \cap \nu_{\frac{G}{H}} \in T F\left(\frac{G}{H}\right)$ and $\mu_{\frac{G}{H}} \cap \nu_{\frac{G}{H}} \subseteq \xi_{\frac{G}{H}}$. If $x H, y H \in \frac{G}{H}$, then

$$
\begin{gathered}
\left(\mu_{\frac{G}{H}} \cap \nu_{\frac{G}{H}}\right)\left(x H y H(x H)^{-1}\right) \\
=T\left(\mu_{\frac{G}{H}}\left(x H y H(x H)^{-1}\right), \nu_{\frac{G}{H}}\left(x H y H(x H)^{-1}\right)\right) \\
\geq T\left(T\left(\mu_{\frac{G}{H}}(y H), \xi_{\frac{G}{H}}(x H)\right), T\left(\nu_{\frac{G}{H}}(y H), \xi_{\frac{G}{H}}(x H)\right)\right) \\
=T\left(T\left(\mu_{\frac{G}{H}}(y H), \nu_{\frac{G}{H}}(y H)\right), T\left(\xi_{\frac{G}{H}}(x H), \xi_{\frac{G}{H}}(x H)\right)\right) \quad(\text { by Lemma 2.5) } \\
=T\left(T\left(\mu_{\frac{G}{H}}(y H), \nu_{\frac{G}{H}}(y H)\right), \xi_{\frac{G}{H}}(x H)\right) \\
=T\left(\left(\mu_{\frac{G}{H}} \cap \nu_{\frac{G}{H}}\right)(y H), \xi_{\frac{G}{H}}(x H)\right) .
\end{gathered}
$$

Therefore $\mu_{\frac{G}{H}} \cap \nu_{\frac{G}{H}} \unrhd \xi_{\frac{G}{H}}$.

Corollary 3.31. Let $I_{n}=\{1,2, \ldots, n\}$ and $\left\{\mu_{i} \mid i \in I_{n}\right\} \subseteq T F\left(\frac{G}{H}\right)$ such that $\left\{\mu_{i} \mid i \in I_{n}\right\} \unrhd \xi$. Then $\mu=\cap_{i \in I_{n}} \mu_{i} \unrhd \xi$. 
FUZZY EQUIVALENCE RELATION, FUZZY CONGRUNCE RELATION AND FUZZY NORMAL SUBGROUPS ON GROUP $G$ OVER

\section{Direct Product of T-FuZzy QUOTIEnT Subgroups AND NORMal $T$-FuZzy QUOTIENT} SUBGROUPS

Definition 4.1. Let $\mu_{\frac{G_{1}}{H_{1}}}$ and $\nu_{\frac{G_{2}}{H_{2}}}$ be $T$-fuzzy subgroups of the groups $\frac{G_{1}}{H_{1}}$ and $\frac{G_{2}}{H_{2}}$, respectively. The direct product of $\mu_{\frac{G_{1}}{H_{1}}}$ and $\nu_{\frac{G_{2}}{H_{2}}}$, denoted by $\mu_{\frac{G_{1}}{H_{1}}} \times \nu_{\frac{G_{2}}{H_{2}}}$, is the function defined by setting for all $x H_{1}$ in $\frac{G_{1}}{H_{1}}$ and $y H_{2}$ in $\frac{G_{2}}{H_{2}}$ we have that

$$
\left(\mu_{\frac{G_{1}}{H_{1}}} \times \nu_{\frac{G_{2}}{H_{2}}}\right)\left(x H_{1}, y H_{2}\right)=T\left(\mu_{\frac{G_{1}}{H_{1}}}\left(x H_{1}\right), \nu_{\frac{G_{2}}{H_{2}}}\left(y H_{2}\right)\right) .
$$

Proposition 4.2. Let $\mu_{i} \in T F\left(\frac{G_{i}}{H_{i}}\right)$ for $i=1,2$. Then $\left(\mu_{1} \times \mu_{2}\right) \in T F\left(\frac{G_{1}}{H_{1}} \times \frac{G_{2}}{H_{2}}\right)$.

Proof. Let $\left(a_{1} H_{1}, b_{1} H_{2}\right),\left(a_{2} H_{1}, b_{2} H_{2}\right) \in \frac{G_{1}}{H_{1}} \times \frac{G_{2}}{H_{2}}$. Then

$$
\begin{gathered}
\left(\mu_{1} \times \mu_{2}\right)\left(\left(a_{1} H_{1}, b_{1} H_{2}\right)\left(a_{2} H_{1}, b_{2} H_{2}\right)\right) \\
=\left(\mu_{1} \times \mu_{2}\right)\left(a_{1} H_{1} a_{2} H_{1}, b_{1} H_{2} b_{2} H_{2}\right) \\
=T\left(\mu_{1}\left(a_{1} H_{1} a_{2} H_{1}\right), \mu_{2}\left(b_{1} H_{2} b_{2} H_{2}\right)\right) \\
\geq T\left(T\left(\mu_{1}\left(a_{1} H_{1}\right), \mu_{1}\left(a_{2} H_{1}\right)\right), T\left(\mu_{2}\left(b_{1} H_{2}\right), \mu_{2}\left(b_{2} H_{2}\right)\right)\right) \\
\geq T\left(\mu_{1}\left(a_{1} H_{1}\right), \mu_{2}\left(b_{1} H_{2}\right), T\left(\mu_{1}\left(a_{2} H_{1}\right), \mu_{2}\left(b_{2} H_{2}\right)\right) \quad(\text { by Lemma 2.5 })\right. \\
=T\left(\left(\mu_{1} \times \mu_{2}\right)\left(a_{1} H_{1}, b_{1} H_{2}\right),\left(\mu_{1} \times \mu_{2}\right)\left(a_{2} H_{1}, b_{2} H_{2}\right)\right) .
\end{gathered}
$$

Also

$$
\begin{gathered}
\left(\mu_{1} \times \mu_{2}\right)\left(a_{1} H_{1}, b_{1} H_{2}\right)^{-1}=\left(\mu_{1} \times \mu_{2}\right)\left(\left(a_{1} H_{1}\right)^{-1},\left(b_{1} H_{2}\right)^{-1}\right) \\
=T\left(\mu_{1}\left(a_{1} H_{1}\right)^{-1}, \mu_{2}\left(b_{1} H_{2}\right)^{-1}\right) \geq T\left(\mu_{1}\left(a_{1} H_{1}\right), \mu_{2}\left(b_{1} H_{2}\right)\right) \\
=\left(\mu_{1} \times \mu_{2}\right)\left(a_{1} H_{1}, b_{1} H_{2}\right) .
\end{gathered}
$$

Thus $\left(\mu_{1} \times \mu_{2}\right) \in T F\left(\frac{G_{1}}{H_{1}} \times \frac{G_{2}}{H_{2}}\right)$.

Corollary 4.3. Let $\mu_{\frac{G_{1}}{H_{1}}} \in T F\left(\frac{G_{1}}{H_{1}}\right)$ and $\nu_{\frac{G_{2}}{H_{2}}} \in T F\left(\frac{G_{2}}{H_{2}}\right)$. Then $\mu_{\frac{G_{1}}{H_{1}}} \times 1_{\frac{G_{2}}{H_{2}}}, 1_{\frac{G_{1}}{H_{1}}} \times \nu_{\frac{G_{2}}{H_{2}}} \in T F\left(\frac{G_{1}}{H_{1}} \times\right.$ $\left.\frac{G_{2}}{H_{2}}\right)$.

Corollary 4.4. Let $\mu_{i} \in T F\left(\frac{G_{i}}{H_{i}}\right)$ for $i=1,2, \ldots, n$. Then

$$
\mu_{1} \times \mu_{2} \times \ldots \times \mu_{n} \in T F\left(\frac{G_{1}}{H_{1}} \times \frac{G_{2}}{H_{2}} \times \ldots \times \frac{G_{n}}{H_{n}}\right) .
$$

Proposition 4.5. Let $\mu_{i} \in N T F\left(\frac{G_{i}}{H_{i}}\right)$ for $i=1,2$. Then $\mu_{1} \times \mu_{2} \in N T F\left(\frac{G_{1}}{H_{1}} \times \frac{G_{2}}{H_{2}}\right)$.

Proof. From Proposition 4.2 we have that $\left(\mu_{1} \times \mu_{2}\right) \in T F\left(\frac{G_{1}}{H_{1}} \times \frac{G_{2}}{H_{2}}\right)$. Let $\left(a_{1} H_{1}, b_{1} H_{2}\right),\left(a_{2} H_{1}, b_{2} H_{2}\right) \in$ $\frac{G_{1}}{H_{1}} \times \frac{G_{2}}{H_{2}}$. Then

$$
\begin{gathered}
\left(\mu_{1} \times \mu_{2}\right)\left(\left(a_{1} H_{1}, b_{1} H_{2}\right)\left(a_{2} H_{1}, b_{2} H_{2}\right)\left(a_{1} H_{1}, b_{1} H_{2}\right)^{-1}\right. \\
=\left(\mu_{1} \times \mu_{2}\right)\left(\left(a_{1} H_{1}, b_{1} H_{2}\right)\left(a_{2} H_{1}, b_{2} H_{2}\right)\left(\left(a_{1} H_{1}\right)^{-1},\left(b_{1} H_{2}\right)^{-1}\right)\right. \\
=\left(\mu_{1} \times \mu_{2}\right)\left(a_{1} H_{1} a_{2} H_{1}\left(a_{1} H_{1}\right)^{-1}, b_{1} H_{2} b_{2} H_{2}\left(b_{1} H_{2}^{-1}\right)\right. \\
=T\left(\mu_{1}\left(a_{1} H_{1} a_{2} H_{1}\left(a_{1} H_{1}\right)^{-1}\right), \mu_{2}\left(b_{1} H_{2} b_{2} H_{2}\left(b_{1} H_{2}\right)^{-1}\right)\right) \\
=T\left(\mu_{1}\left(a_{2} H_{1}\right), \mu_{2}\left(b_{2} H_{2}\right)\right) \\
=\left(\mu_{1} \times \mu_{2}\right)\left(a_{2} H_{1}, b_{2} H_{2}\right) .
\end{gathered}
$$


Corollary 4.6. Let $\mu_{i} \in N T F\left(\frac{G_{i}}{H_{i}}\right)$ for $i=1,2, \ldots, n$. Then

$$
\mu_{1} \times \mu_{2} \times \ldots \times \mu_{n} \in N T F\left(\frac{G_{1}}{H_{1}} \times \frac{G_{2}}{H_{2}} \times \ldots \times \frac{G_{n}}{H_{n}}\right) .
$$

Proposition 4.7. Let $\mu_{i}, \nu_{i} \in T F\left(\frac{G_{i}}{H_{i}}\right)$ and $\mu_{i} \subseteq \nu_{i}$ for $i=1$, 2 . If $\mu_{i} \unrhd \nu_{i}$, then $\mu_{1} \times \mu_{2} \unrhd \nu_{1} \times \nu_{2}$. Proof. Let $\left(a_{1} H_{1}, b_{1} H_{2}\right),\left(a_{2} H_{1}, b_{2} H_{2}\right) \in \frac{G_{1}}{H_{1}} \times \frac{G_{2}}{H_{2}}$. Then

$$
\begin{gathered}
\left(\mu_{1} \times \mu_{2}\right)\left(\left(a_{1} H_{1}, b_{1} H_{2}\right)\left(a_{2} H_{1}, b_{2} H_{2}\right)\left(a_{1} H_{1}, b_{1} H_{2}\right)^{-1}\right) \\
=\left(\mu_{1} \times \mu_{2}\right)\left(\left(a_{1} H_{1}, b_{1} H_{2}\right)\left(a_{2} H_{1}, b_{2} H_{2}\right)\left(\left(a_{1} H_{1}\right)^{-1},\left(b_{1} H_{2}\right)^{-1}\right)\right) \\
=\left(\mu_{1} \times \mu_{2}\right)\left(a_{1} H_{1} a_{2} H_{1}\left(a_{1} H_{1}\right)^{-1}, b_{1} H_{2} b_{2} H_{2}\left(b_{1} H_{2}\right)^{-1}\right) \\
=T\left(\mu_{1}\left(a_{1} H_{1} a_{2} H_{1}\left(a_{1} H_{1}\right)^{-1}\right), \mu_{2}\left(b_{1} H_{2} b_{2} H_{2}\left(b_{1} H_{2}\right)^{-1}\right)\right) \\
\geq T\left(T\left(\mu_{1}\left(a_{2} H_{1}\right), \nu_{1}\left(a_{1} H_{1}\right)\right), T\left(\mu_{2}\left(b_{2} H_{2}\right), \nu_{2}\left(b_{1} H_{2}\right)\right)\right) \\
=T\left(T\left(\mu_{1}\left(a_{2} H_{1}\right), \mu_{2}\left(b_{2} H_{2}\right)\right), T\left(\nu_{1}\left(a_{1} H_{1}\right), \nu_{2}\left(b_{1} H_{2}\right)\right)\right) \quad(\text { by Lemma 2.5 }) \\
=T\left(\left(\mu_{1} \times \mu_{2}\right)\left(a_{2} H_{1}, b_{2} H_{2}\right),\left(\nu_{1} \times \nu_{2}\right)\left(a_{1} H_{1}, b_{1} H_{2}\right)\right) .
\end{gathered}
$$

Corollary 4.8. Let $\mu_{i}, \nu_{i} \in T F\left(\frac{G_{i}}{H_{i}}\right)$ and $\mu_{i} \subseteq \nu_{i}$ for $i=1,2, \ldots, n$. If $\mu_{i} \unrhd \nu_{i}$, then $\mu_{1} \times \mu_{2} \times \ldots \times \mu_{n} \unrhd \nu_{1} \times \nu_{2} \times \ldots \times \nu_{n}$.

5. GROUP HOMOMORPHISMS AND FUZZY SUBGROUPS UNDER $t$-NORMS

Lemma 5.1. Let $f$ be a homomorphism of $\frac{G_{1}}{H_{1}}$ into $\frac{G_{2}}{H_{2}}$ and $\mu \in T F\left(\frac{G_{1}}{H_{1}}\right)$. Then $f(\mu) \in T F\left(\frac{G_{2}}{H_{2}}\right)$.

Proof. Let $u H_{2}, v H_{2} \in \frac{G_{2}}{H_{2}}$ and $x H_{1}, y H_{1} \in \frac{G_{1}}{H_{1}}$. If $u H_{2} \notin f\left(\frac{G_{1}}{H_{1}}\right)$ or $v H_{2} \notin f\left(\frac{G_{1}}{H_{1}}\right)$, then

$$
f(\mu)\left(u H_{2}\right)=f(\mu)\left(v H_{2}\right)=0 \leq f(\mu)\left(u H_{2} v H_{2}\right) .
$$

Suppose $u H_{2}=f\left(x H_{1}\right)$ and $v H_{2}=f\left(y H_{1}\right)$ then

$$
\begin{gathered}
f(\mu)\left(u H_{2} v H_{2}\right) \\
=\sup \left\{\mu\left(x H_{1} y H_{1}\right) \mid u H_{2}=f\left(x H_{1}\right), v H_{2}=f\left(y H_{1}\right)\right\} \\
\geq \sup \left\{T\left(\mu\left(x H_{1}\right), \mu\left(y H_{1}\right)\right) \mid u H_{2}=f\left(x H_{1}\right), v H_{2}=f\left(y H_{1}\right)\right\} \\
=T\left(\sup \left\{\mu\left(x H_{1}\right) \mid u H_{2}=f\left(x H_{1}\right)\right\}, \sup \left\{\mu\left(y H_{1}\right) \mid v H_{2}=f\left(y H_{1}\right)\right\}\right) \\
=T\left(f(\mu)\left(u H_{2}\right), f(\mu)\left(v H_{2}\right)\right) .
\end{gathered}
$$

Also since $\mu \in T F\left(\frac{G_{1}}{H_{1}}\right)$ we have

$$
\begin{gathered}
f(\mu)\left(\left(u H_{2}\right)^{-1}\right)=f(\mu)\left(\left(u^{-1} H_{2}\right)\right) \\
=\sup \left\{\mu\left(x H_{1}\right) \mid u^{-1} H_{2}=f\left(x H_{1}\right)\right\} \\
\geq \sup \left\{\mu\left(x^{-1} H_{1}\right) \mid u H_{2}=f\left(x^{-1} H_{1}\right)\right\} \\
=f(\mu)\left(u H_{2}\right) .
\end{gathered}
$$

Lemma 5.2. Let $f$ be a homomorphism of $\frac{G_{1}}{H_{1}}$ into $\frac{G_{2}}{H_{2}}$ and $\nu \in T F\left(\frac{G_{2}}{H_{2}}\right)$. Then $f^{-1}(\nu) \in$ $T F\left(\frac{G_{1}}{H_{1}}\right)$. 
FUZZY EQUIVALENCE RELATION, FUZZY CONGRUNCE RELATION AND FUZZY NORMAL SUBGROUPS ON GROUP $G$ OVER

Proof. Let $x H_{1}, y H_{1} \in \frac{G_{1}}{H_{1}}$. Then

$$
\begin{gathered}
f^{-1}(\nu)\left(x H_{1} y H_{1}\right)=\nu\left(f\left(x H_{1} y H_{1}\right)\right)=\nu\left(f\left(x H_{1}\right) f\left(y H_{1}\right)\right. \\
\geq T\left(\nu\left(f\left(x H_{1}\right)\right), \nu\left(f\left(y H_{1}\right)\right)\right)=T\left(f^{-1}(\nu)\left(x H_{1}\right), f^{-1}(\nu)\left(y H_{1}\right)\right) .
\end{gathered}
$$

Also

$f^{-1}(\nu)\left(x H_{1}\right)^{-1}=\nu\left(f\left(x H_{1}\right)^{-1}\right)=\nu\left(f^{-1}\left(x H_{1}\right)\right) \geq \nu\left(f\left(x H_{1}\right)\right)=f^{-1}(\nu)\left(x H_{1}\right)$.

Proposition 5.3. Let $\mu \in N T F\left(\frac{G_{1}}{H_{1}}\right)$ and $f$ is an epimorphism of $\frac{G_{1}}{H_{1}}$ onto $\frac{G_{2}}{H_{2}}$. Then $f(\mu) \in$ $N T F\left(\frac{G_{2}}{H_{2}}\right)$.

Proof. From Lemma 5.1 we have $f(\mu) \in T F\left(\frac{G_{2}}{H_{2}}\right)$. Let $x H_{2}, y H_{2} \in \frac{G_{2}}{H_{2}}$. Since $f$ is a surjection, $f\left(u H_{1}\right)=x H_{2}$ for some $u H_{1} \in \frac{G_{1}}{H_{1}}$. Then

$$
\begin{gathered}
f(\mu)\left(x H_{2} y H_{2}\left(x H_{2}\right)^{-1}\right) \\
=\sup \left\{\mu\left(w H_{1}\right) \mid w H_{1} \in \frac{G_{1}}{H_{1}}, f\left(w H_{1}\right)=x H_{2} y H_{2}\left(x H_{2}\right)^{-1}\right\} \\
=\sup \left\{\mu\left(u^{-1} H_{1} w H_{1} u H_{1}\right) \mid\right. \\
\left.w H_{1} \in \frac{G_{1}}{H_{1}}, f\left(u^{-1} H_{1} w H_{1} u H_{1}\right)=y H_{2}\right\} \\
=f(\mu)\left(y H_{2}\right) .
\end{gathered}
$$

Proposition 5.4. Let $\frac{G_{2}}{H_{2}}$ be a group and $\nu \in N T F\left(\frac{G_{1}}{H_{1}}\right)$. Suppose that $f$ is a homomorphism of $\frac{G_{1}}{H_{1}}$ into $\frac{G_{2}}{H_{2}}$. Then $f^{-1}(\nu) \in N T F\left(\frac{G_{1}}{H_{1}}\right)$.

Proof. By Lemma 5.2 we obtain $f^{-1}(\nu) \in T F\left(\frac{G_{1}}{H_{1}}\right)$. Now for any $x H_{1}, y H_{1} \in \frac{G_{1}}{H_{1}}$, we have

$$
\begin{aligned}
& f^{-1}(\nu)\left(x H_{1} y H_{1}\left(x H_{1}\right)^{-1}\right) \\
= & \nu\left(f\left(x H_{1} y H_{1}\left(x H_{1}\right)^{-1}\right)\right) \\
= & \nu\left(f\left(x H_{1}\right) f\left(y H_{1}\right) f\left(x H_{1}\right)^{-1}\right) \\
= & \nu\left(f\left(x H_{1}\right) f\left(y H_{1}\right) f^{-1}\left(x H_{1}\right)\right) \\
= & \nu\left(f\left(y H_{1}\right)\right)=f^{-1}(\nu)\left(y H_{1}\right) .
\end{aligned}
$$

Hence $f^{-1}(\nu) \in N T F\left(\frac{G_{1}}{H_{1}}\right)$.

Proposition 5.5. Let $\mu, \nu \in T F\left(\frac{G_{1}}{H_{1}}\right)$ and $\mu \unrhd \nu$. Let $\frac{G_{2}}{H_{2}}$ be a group and $f$ a homomorphism from $\frac{G_{1}}{H_{1}}$ into $\frac{G_{2}}{H_{2}}$. Then $f(\mu) \unrhd f(\nu)$.

Proof. By Lemma 5.1 we have $f(\mu), f(\nu) \in T F\left(\frac{G_{2}}{H_{2}}\right)$. Let $x H_{2}, y H_{2} \in \frac{G_{2}}{H_{2}}$ and $u H_{1}, v H_{1} \in \frac{G_{1}}{H_{1}}$. Then

$$
\begin{gathered}
f(\mu)\left(x H_{2} y H_{2}\left(x H_{2}\right)^{-1}\right) \\
=\sup \left\{\mu\left(z H_{1}\right) \mid z H_{1} \in \frac{G_{1}}{H_{1}}, f\left(z H_{1}\right)=x H_{2} y H_{2}\left(x H_{2}\right)^{-1}\right\} \\
=\sup \left\{\mu\left(u H_{1} v H_{1}\left(u H_{1}\right)^{-1}\right) \mid u H_{1}, v H_{1} \in \frac{G_{1}}{H_{1}}, f\left(u H_{1}\right)=x H_{2}, f\left(v H_{1}\right)=y H_{2}\right\} \\
\geq \sup \left\{T\left(\mu\left(v H_{1}\right), \nu\left(u H_{1}\right)\right) \mid f\left(u H_{1}\right)=x H_{2}, f\left(v H_{1}\right)=y H_{2}\right\} \\
=T\left(\sup \left\{\mu\left(v H_{1}\right) \mid y H_{2}=f\left(v H_{1}\right)\right\}, \sup \left\{\nu\left(u H_{1}\right) \mid x H_{2}=f\left(u H_{1}\right)\right\}\right) \\
=T\left(f(\mu)\left(y H_{2}\right), f(\nu)\left(x H_{2}\right)\right) .
\end{gathered}
$$


Hence $f(\mu) \unrhd f(\nu)$.

Proposition 5.6. Let $\frac{G_{2}}{H_{2}}$ be a group. Let $\mu, \nu \in T F\left(\frac{G_{2}}{H_{2}}\right)$ and $\mu \unrhd \nu$. If $f$ be a homomorphism from $\frac{G_{1}}{H_{1}}$ into $\frac{G_{2}}{H_{2}}$, then $f^{-1}(\mu) \unrhd f^{-1}(\nu)$.

Proof. From Lemma 5.2 we have $f^{-1}(\mu), f^{-1}(\nu) \in T F\left(\frac{G_{1}}{H_{1}}\right)$. Let $x H_{1}, y H_{1} \in \frac{G_{1}}{H_{1}}$. Now

$$
\begin{gathered}
f^{-1}(\mu)\left(x H_{1} y H_{1}\left(x H_{1}\right)^{-1}\right)=\mu\left(f\left(x H_{1} y H_{1}\left(x H_{1}\right)^{-1}\right)\right) \\
=\mu\left(f\left(x H_{1}\right) f\left(y H_{1}\right) f^{-1}\left(x H_{1}\right)\right) \geq T\left(\mu\left(f\left(y H_{1}\right)\right), \nu\left(f\left(x H_{1}\right)\right)\right) \\
=T\left(f^{-1}(\mu)\left(y H_{1}\right), f^{-1}(\nu)\left(x H_{1}\right)\right) .
\end{gathered}
$$

Hence $f^{-1}(\mu) \unrhd f^{-1}(\nu)$.

Acknowledgment. We would like to thank the referees for carefully reading the manuscript and making several helpful comments to increase the quality of the paper.

\section{REFERENCES}

[1] M. T. Abu Osman, On some products of fuzzy subgroups, Fuzzy Sets and Systems, 24 (1987), 79-86.

[2] J. M. Anthony and H. Sherwood, Fuzzy groups redefined, J. Math. Anal. Appl., 69(1979), 124-130.

[3] D. R. Bae and J. P. Kim, Fuzzy congruences in groups, Fuzzy Sets and Systems, 85(1997), 115-120.

[4] I. Beg, Fuzzy multivalued functions, Bull. Allahabad Math. Soc., 21(2006), 41-104.

[5] I. Beg and S. Ashraf, Fuzzy similarity and measure of similarity with Lukasiewicz implicator, New Math. Natural Computation, 4(2008), 191-206.

[6] I. Beg and S. Ashraf, Fuzzy inclusion and fuzzy similarity with Godel implication operator, New Math. Natural Computation, 5(2009), 617-633.

[7] I. Beg and S. Ashraf, Fuzzy dissimilarity measures and distance functions, Fuzzy Information and Engineering, 1(2009), 205-217.

[8] I. Beg and S. Ashraf, Similarity measures for fuzzy sets, Appl. and Comput. Math., 8(2009), 192-202.

[9] I. Beg and S. Ashraf, Fuzzy Relations, Lambert Academic Publisher, Germany, 2009.

[10] V. V. Cross and T. A. Sudkamp, Similarity and Compatibility in Fuzzy Set Theory, Springer-Verlag, Heidelberg 2002.

[11] D. Dubois and H. Prade, Fundamentals of Fuzzy Sets, Kluwer Academic Publishers, Dordrecht - 2000.

[12] S. Gottwald, Fuzzy Sets and Fuzzy Logic, Vieweg, Wiesbaden, 1993.

[13] U. Hohle and S. E. Rodabaugh, Mathematics of Fuzzy Sets: Logic, Topology, and Measure Theory, The Hand book of Fuzzy Mathematics Series, 1998.

[14] E. E. Kerre, Introduction to the Basic Principles of Fuzzy Set Theory and some of its Applications, Communication and Cognition, 1991.

[15] E. E. Kerre, Basic principles of fuzzy set theory for the representation and manipulation of imprecision and uncertainty, Communication and Cognition, Gent, second revised edition (1993), 1-158.

[16] E. Kerre, An overview of fuzzy relational calculus and its applications, Lecture Notes in Computer Science, 4617(2007), 1-13.

[17] I. J. Kumar, P. K. Saxena and P. Yadava, Fuzzy normal subgroups and fuzzy quotients, Fuzzy Sets and Systems, 46(1992), 121-132.

[18] N. Kuroki, Fuzzy congruences and Fuzzy normal subgroups, Inform. Sci., 60(1992), 247-361.

[19] H. X. Li and V. C. Yen, Fuzzy Sets and Fuzzy Decision-Making, CRC Press. Inc., London, 1995.

[20] D. S. Malik and J. N. Mordeson, A note on fuzzy relations and fuzzy groups, Inform. Sci., 56(1991), 193-198.

[21] K. Menger, Probabilistic theories of relations, Proc. Nat. Acad. Sci. USA, 37 (1951), 178-180.

[22] C. V. Negoita and D. A. Ralescu, Representation theorems for fuzzy concepts, Kybernetes, 4(1975), 169-174.

[23] H. T. Nguyen and E. A. Walker, Fuzzy Logic, CRC Press. Boston, 2006.

[24] K. Peeva and Y. Kyosev, Fuzzy Relational Calculus, World Scientific, New Jersey 2004.

[25] H. Poincare, La Science et Hypothese, Flammarion, Paris, 1902.

[26] R. Rasuli, Fuzzy Ideals of Subtraction Semigroups with Respect to A t-norm and A t-conorm, The Journal of Fuzzy Mathematics Los Angeles, 24 (4) (2016), 881-892. 
[27] R. Rasuli, Fuzzy modules over a t-norm, Int. J. Open Problems Compt. Math., 9 (3) (2016), 12-18.

[28] R. Rasuli, Fuzzy Subrings over a t-norm, The Journal of Fuzzy Mathematics Los Angeles, 24 (4) (2016), 995-1000.

[29] R. Rasuli, Norms over intuitionistic fuzzy subrings and ideals of a ring, Notes on Intuitionistic Fuzzy Sets, 22 (5) (2016), 72-83.

[30] R. Rasuli, Norms over fuzzy Lie algebra, Journal of New Theory, 15(2017), 32-38.

[31] R. Rasuli, Fuzzy subgroups on direct product of groups over a t-norm, Journal of Fuzzy Set Valued Analysis, 3(2017), 96-101.

[32] R. Rasuli, Characterizations of intuitionistic fuzzy subsemirings of semirings and their homomorphisms by norms, Journal of New Theory, 18(2017), 39-52.

[33] R. Rasuli, Intuitionistic fuzzy subrings and ideals of a ring under norms, LAP LAMBERT Academic publishing, 2017, ISBN: 978-620-2-06926-7.

[34] R. Rasuli, Characterization of Q-Fuzzy subrings (Anti Q-Fuzzy Subrings) with respect to a T-norm (TConorms), Journal of Information and Optimization Science, 31(2018), 1-11.

[35] R. Rasuli, T-Fuzzy Submodules of $R \times M$, Journal of New Theory, 22(2018), 92-102.

[36] R. Rasuli, Fuzzy subgroups over a T-norm, Journal of Information and Optimization Science, 39(2018), 1757-1765.

[37] A. Rosenfeld, Fuzzy Groups, J. Math. Anal. Appl., 35(1971), 512-517.

[38] B. Russell, Vagueness, Australian J. Phil., 1(1923), 84-92.

[39] M. Samhan, Fuzzy congruences on semigroups, Inform. Sci., 74(1993), 165-175.

[40] L. A. Zadeh, Similarity relations and fuzzy orderings, Inf. Sci., 3(1971), 177-200.

[41] L. A. Zadeh, Fuzzy Set Theory and its Applications, Kluwer Academic Publishers, Boston, 1991.

[42] L. A. Zadeh, Fuzzy sets, Inform. Control., 8(1965), 338-353.

[43] L. A. Zadeh, Generalized theory of uncertainty (GTU)principal concepts and ideas, Comput. Statist. Data Anal., 51(2006), 15-46.

[44] L. A. Zadeh, How do you define similarity?, Private Communication, 2007.

Department of Mathematics, Payame Noor University (PNU), Tehran, Iran.

E-mail address: rasulirasul@yahoo.com 